\title{
Building on Julian Tudor Hart's example of anticipatory care
}

\author{
Graham Watt ${ }^{1}$, Catherine O'DonnelI $^{2}$ and Sanjeev Sridharan ${ }^{3,4}$ \\ ${ }^{1}$ Professor of General Practice, General Practice and Primary Care, Division of Community-Based Sciences, \\ University of Glasgow, Glasgow, UK \\ ${ }^{2}$ Professor of Primary Care Research and Development, Division of Community-Based Sciences, University of \\ Glasgow, Glasgow, UK \\ ${ }^{3}$ Director, Evaluation, The Centre for Research in Inner City Health, The Keenan Research Centre, Li Ka Shing \\ Knowledge Institute, St Michael's Hospital, Toronto, Ontario, Canada \\ ${ }^{4}$ Associate Professor, Health Policy, Management and Evaluation, University of Toronto, Toronto, Ontario, Canada
}

\begin{abstract}
The prevention and delay of chronic disease is an increasing priority in all advanced health-care systems, but sustainable, effective and equitable approaches remain elusive. In a famous pioneering example in the UK, Julian Tudor Hart combined reactive and anticipatory care within routine consultations in primary medical care, while applying a population approach to delivery and audit. This approach combined the structural advantages of UK general practice, including universal coverage and the absence of user fees, with his long-term commitment to individual patients, and was associated with a $28 \%$ reduction in premature mortality over a 25 -year period. The more recent, and comprehensively evaluated Scottish National Health Service demonstration project, 'Have a Heart Paisley', took a different approach to cardiovascular prevention and health improvement, using population screening for ascertainment, health coaches and referral to specific health improvement programmes for diet, smoking and exercise. We draw from both examples to construct a conceptual framework for anticipatory care, based on active ingredients, programme pathways and whole system approaches. While the strengths of a family practice approach are coverage, continuity, co-ordination and longterm relationships, the larger health improvement programme offered additional resources and expertise. As theory and evidence accrue, the challenge is to combine the strengths of primary medical care and health improvement, in integrated, sustainable systems of anticipatory care, addressing the heterogeneity of individual needs and solutions, while achieving high levels of coverage, continuity, co-ordination and outcome.
\end{abstract}

Key words: anticipatory care; family medicine; primary care

Received 25 February 2010; accepted 30 May 2010; first published online 12 August 2010

\section{Introduction}

The prevention and delay of chronic disease is an increasing priority in all health-care systems. The challenge is complicated by increasing morbidity, widening inequity and spiralling costs (World Health Organization, 2008). As Stange (2009)

Correspondence to: Professor Graham Watt, General Practice and Primary Care, Division of Community-based Sciences, University of Glasgow, 1 Horselethill Road, Glasgow G11 5AW, UK. Email: gcmw1j@clinmed.gla.ac.uk

(C) Cambridge University Press 2010 cogently argues, part of the problem in addressing these challenges is the increasing fragmentation of care, with a lack of integration between specialists and generalists, and between preventive activity and reactive activity, with increasing depersonalization of care, regardless of the health system.

In developed societies with organized health services dedicated to improving population health, a major task is to complement reactive care, responding to health problems after they have occurred, with an anticipatory approach concerned with the prevention of future problems (Scottish Executive, 2005; 
Scottish Government, 2007). To use a familiar metaphor, anticipatory care is an upstream activity that tries to prevent, delay or lessen the severity of downstream events.

In this paper, we first consider what is meant by anticipatory care and then draw from two substantial practical examples in the UK - the original example of Julian Tudor Hart and the recent Scottish National Health Service (NHS) demonstration project, 'Have a Heart Paisley'. The development of effective, equitable and sustainable models of anticipatory care can learn from both examples. To take the matter forward, we propose a taxonomy of system development for primary care, drawing on theory, evidence and practice to identify the key issues that developments in anticipatory care must address (Craig et al., 2008; Murray and Frank, 2008).

\section{What is anticipatory care?}

Anticipatory care may be defined broadly as health improvement activities, including primary, secondary and tertiary prevention, which are delivered within or in association with health care, and involving direct contact with target populations. A wide range of health care and health improvement activities are included by this definition, while many aspects of health improvement policy and health promotion that do not require an association with health care are not. The successful outcome of anticipatory care is that individuals adopt and maintain one or more behaviours concerned with their future health and well-being, thus avoiding, reducing or preventing disease risks and complications.

Anticipatory care is clearly not the only way in which population health may be improved, but it is the principal way in which population health can be improved by health professionals working in primary care.

\section{Experiences of anticipatory care in general practice}

Two pioneers of anticipatory care were the Dutch general practitioner Van den Dool, who invented the term, and the British general practitioner, Julian Tudor Hart, who followed his example in the late 1960s.

Primary Health Care Research \& Development 2011; 12: 3-10
Van den Dool collaborated with a university department of general practice to screen all his patients for their disease risks (Van den Dool, 1973). After five years, this information became out of date, so the screening process was repeated. Van den Dool realized that this approach was unsustainable and could not be applied to large number of practices. From then on he ascertained the health risks of his population in a different way, using the routine contacts that he had with the large majority of his patients (typically involving over $90 \%$ of patients over a five-year period) to collect the information on a prospective and cumulative basis.

Julian Tudor Hart applied this approach at Glyncorrwg in South Wales, where he became the first general practitioner in the world to measure the blood pressure of all his patients (Hart, 1970). This was not so much an epidemiological task, as the practical expression of a philosophy of primary care. At a conference in Glasgow in 2007 to celebrate Julian Tudor Hart's 80th birthday, his contribution as a general practitioner in the British NHS was described as:

\section{the combination of a population approach with long term productive relationships, between patients and professionals who know and trust each other, and who are guided by evidence and audit, is a powerful force not only for epidemiological research, but also for health improvement, and a fairer, more convivial society.}

Tudor Hart's example is important because it was unusually well documented over a 25-year period and was associated with substantial health benefits for the population served (Hart et al., 1991). Compared with a neighbouring population, with similar socio-economic characteristics but receiving conventional care, premature mortality was $28 \%$ lower in the population that had received Tudor Hart's model of anticipatory care. The example has influenced NHS policy, especially in Scotland, where anticipatory care is intended to "enable the NHS to play a full part in promoting good heath ...., giving greater emphasis to preventive medicine and earlier intervention ('anticipatory care'), especially in areas with the poorest health' (Scottish Executive, 2005; Scottish Government, 2007). The approach is also apparent in the development of a 
more holistic approach to health in Wales and in the introduction of 'Life Checks' in England.

Tudor Hart worked in a small, relatively isolated and socio-economically deprived community of about 1500 people in South Wales between 1961 and 1987, and because of this particular setting in time and place, his example is not necessarily a model that can be applied now or elsewhere. However, it does represent the origin of many mainstream features of current primary care.

\section{Key components of the Tudor Hart approach: the centrality of routine encounters}

Tudor Hart's approach to anticipatory care was to find time within routine consultations to deal not only with today's problems as presented by patients, but also to address future problems. This patient-centred approach included a wide range of preventive activities, including the reversal of risks in people who were otherwise well and the prevention of complications in people with established conditions. Most attention focused on the risks of high blood pressure, smoking, alcohol, diabetes and obesity - the possibilities of cholesterol-lowering not being established until the late 1980s.

By combining anticipatory and reactive care within routine consultations, there was little need to establish separate contacts with patients for preventive activity. This removed the need to consider whether all patients in the practice should be approached or whether such activity should be restricted to those deemed at high risk. Over several years these routine contacts with the general practitioner and practice nurses provided a high level of population coverage. Special measures for making contact, such as knocking on doors, were only required for a minority of patients (Hart, 1993).

By addressing patient's immediate concerns first, there was a greater possibility of engaging their interest in other issues, such as changes in behaviour to avoid, delay or reduce the severity of future health problems. Established relationships and trust, built through serial routine consultations, provided the continuity and flexibility required not only to work through problems and challenges but also to address issues in the order, and at the pace, which suited patients. Anticipatory care, in this context, involved a shift from the traditional role of patients as passive consumers to active participants in their own care, 'co-producing' their health in collaboration with health professionals (Hart, 2006).

\section{Key components of the Tudor Hart approach: building a system}

When Tudor Hart first set out to measure the blood pressures of all his patients, he began by screening the records, not the patients, to establish what had already been done, to identify what needed to be done and to flag the records so that practice staff would be alerted to these outstanding tasks the next time the patient chose to visit the practice.

The patients for whom continuity and flexibility were most important were those with multiple problems, who are most at risk of receiving fragmented care from multiple providers and who most need coherent help and guidance. In the Tudor Hart example, about half of hypertensive patients attended a dedicated blood pressure clinic; the other half had other major problems in addition to high blood pressure and were best served within routine consultations (Hart, 1993).

The contribution to population health of this approach depended not only on the availability of specific interventions of proven effectiveness and their incorporation in productive, long-term patient-doctor relationships, but also their delivery to large numbers of people. The necessary practice infrastructure included the development of high-quality clinical records, the involvement of the practice receptionist and nurse as key colleagues, regular audit to identify not only what had been achieved, but also what had not been achieved - the 'measurement of omission' (Hart, 1982) - and the flagging of records to identify outstanding tasks. This systematic approach is much easier to deliver in the 21st century, particularly in systems making effective use of electronic medical records.

\section{Limitations of the Tudor Hart model}

A feature of the Tudor Hart approach was that almost all aspects of anticipatory care were integrated

Primary Health Care Research \& Development 2011; 12: 3-10 
within a single general practice, with little availability of, or linkage to specific health improvement programmes, such as dietary advice, smoking cessation or exercise referral. As populations get older, with increasing needs and demands for health care, it is increasingly difficult for small practices to deliver the full range of services that patients require.

A key feature was the steady, cumulative approach over a long period of time. The benefits of such an approach may be difficult to detect in the short term, especially if they accrue like compound interest towards the end of a long period. Continuity of care over such a period is an increasing challenge, requiring effective communication between professional colleagues and productive use of written and electronic records (Freeman et al., 2003).

Tudor Hart's approach utilized aspects of the UK NHS, which may not apply in other health systems, including universal access and the absence of user fees. However, although such features may be considered desirable, they are not essential. The general approach can be applied in any clinic population. In the UK primary-care system, population coverage is achieved largely via the sum of what is provided for clinic populations. Although user fees can make it difficult for practitioners to introduce issues in the consultation, which have not been raised by the patient, many aspects of anticipatory care flow directly from the wider management and prevention of presented problems.

\section{Evidence from a case study: Have a Heart Paisley}

A more recent, contrasting and larger example of anticipatory care is 'Have a Heart Paisley', the Scottish NHS demonstration project (2001-2008), in which 11277 people aged 45-60 years, comprising the entire population in this age group in a large Scottish town served by 13 general practices, were offered an initial cardiovascular and general health risk assessment, with referral to associated health improvement programmes for smoking, diet and exercise, with support for individuals from dedicated health coaches. These types of support were also made available for the secondary prevention of health problems in patients

Primary Health Care Research \& Development 2011; 12: 3-10 with established heart disease. The mixed method evaluation of this large and complex intervention revealed the following findings (Sridharan et al., 2008).

- Engagement with the programme was inversely related to individual deprivation. Men were less likely to engage than women. Health coaches felt that the recruitment strategy used had largely failed to engage with the target 'hard-toreach' population and had resulted in an overrepresentation of the worried well.

- The main defining feature for clients was that the health coach had time for engagement, which contrasted with their perception of other health professionals, especially doctors. Some participants felt a continued need for support after the programme had finished, and that a year may not be sufficient to make behavioural changes that could be sustained in the longer term.

- The primary prevention was perhaps more likely to work for motivated individuals, who wanted to change but needed both the encouragement and the education to know how to follow through.

- Flexibility was frequently mentioned as an important and inherent characteristic of the health-coaching model, in terms of time of meetings, location and setting of goals and interaction with coaches. Coaches also reported that clients frequently had unresolved mental health or alcohol related issues, which sometimes needed to be addressed as a pre-requisite to being able to work on the three topic areas of exercise, diet and smoking.

- Implementation of the secondary prevention programme was limited by low levels of referral from primary care, which were attributed to the absence of key players from primary care at the planning stage.

The experience of 'Have a Heart Paisley' illustrates that in devising new systems of anticipatory care, which aim to target a wide population base, several challenges have to be overcome (Sridharan et al., 2008).

Approaches to population coverage that involve episodic screening can generate large number of contacts, but it is important to review the characteristics of both those who respond to such invitations and those who do not. A key 
question is whether the approach includes individuals who have not previously been in contact with services - the genuinely 'hard to reach'. In view of the high cumulative population coverage achieved by routine general practice in the UK, it is likely that many apparently 'hard-to-reach' individuals are, in fact, contact with services, but that these contacts are not used for anticipatory care.

Ascertainment of large numbers of people at the start of an anticipatory care initiative results in large caseloads for newly recruited staff, not only putting pressure on these resources but also raising questions about long-term sustainability. The less-intense Tudor Hart approach, with no pressure to generate or process large number of individuals at any one time, is more sustainable, especially when delivered through existing services. On the other hand, centrally organized resources may be the most efficient way of providing specialist resources that individual general practices cannot provide.

When individuals have a range of health and associated problems, the challenge is to help co-ordinate their use of resources, in an order and at a pace that is suitable to them, while maintaining confidence and continuity. Given the central importance for some individuals of longterm productive relationships with a health professional, and the different ways in which such relationships can be established and maintained (eg, with health coaches, general practitioners, community nurses), it is important to encourage diversity within arrangements for anticipatory care, while retaining the ability to review and audit the system as a whole.

\section{Comparing population approaches}

We have chosen these two examples not only because they were unusually well documented but also because they help to illustrate different and complementary approaches to population care. The contrast is not limited to their different approaches to ascertainment.

'Have a Heart Paisley' is typical of the high-risk strategy of prevention, in which a population is systematically screened to identify high-risk individuals who can benefit from specific interventions. Such approaches only involve population contact at the outset, and are often contrasted with mass strategies of prevention, which aim to influence the health behaviours of a population without direct contact (Rose, 1985).

Tudor Hart's approach to anticipatory care combined the high-risk and mass approaches, in a process of continual engagement with the population, working flexibly to address individual patients' needs and problems - in effect, combining many high-risk strategies within a single integrated system serving a defined population and addressing patients' needs over a long period of time. In the next section, we attempt to identify the transferable elements of his approach.

\section{Developing a theory of system development}

Anticipatory care can be described as 'complex systems thrust upon complex systems' (Pawson, 2006). Three useful concepts in identifying and considering anticipatory care are active ingredients, programme pathways and the heterogeneity of individual needs.

\section{Active ingredients}

By active ingredients, we mean the elements of the intervention, which bring about change in behaviour by an individual. Defining the active ingredient of a 'simple' intervention such as prescribing aspirin, or giving health advice, is easier than defining the active ingredients of a complex social intervention such as anticipatory care (Pawson, 2006). The active ingredients of anticipatory care can be considered as serial measures designed to achieve:

- Initial contact

- Risk assessment

- Explanation and discussion of options

- Agreement on a course of action

- Referral to expert help, when indicated

- Maintenance of the course of action

- Co-ordination of multiple problems

- Follow up and review.

\section{Programme pathways}

We define programme pathways simply as a process by which an intervention can deliver the

Primary Health Care Research \& Development 2011; 12: 3-10 
active ingredients. It is axiomatic that the active ingredients of anticipatory care can be delivered via more than one type of pathway. Anticipatory care typically involves much planning, targeting, reaching, screening and empowering of patients to ensure that the active ingredients can be triggered.

The key aspect of anticipatory care, which distinguishes it from high-quality, holistic, personal care of individual patients, is the planning, delivery and review of this work from a population perspective. Audit is the key to ensure that evidence is applied not just for individual patients who happen to attend, but for all patients who can benefit.

Systems of anticipatory care should include and be audited to review the following features:

- Coverage - what proportion and sections of the target population have taken part?

- Continuity - have participants proceeded through each of the steps of anticipatory care?

- Co-ordination - do the component parts of anticipatory care work effectively and efficiently together?

- Balance - does the system maintain an appropriate balance, and investment of resources, between the serial stages of anticipatory care?

- Sustainability - have arrangements been established to maintain anticipatory care for the long term?

In focusing on the population, the challenge is not only to deliver the individual components of anticipatory care (concerned with smoking, diet; alcohol, exercise, psychological distress, etc.), but also to combine and maintain these activities for individual patients, especially when they have more than one problem.

\section{Heterogeneity of needs and mechanisms}

Individuals may need different kinds of approach to trigger the active ingredients, simply because they have different social and individual needs and constraints. An example of heterogeneity in planning a small part of anticipatory care is related to reaching individuals to ascertain needs. For some individuals, a mailed letter might suffice; for some, an advertisement on the radio; others will need a recommendation from a close friend or family member; others will need more concerted efforts and some may not respond to

Primary Health Care Research \& Development 2011; 12: 3-10 any type of approach and only be contactable when they attend for another reason.

Heterogeneity is also apparent in how people respond to anticipatory care. Some are capable of addressing their future needs independently of health professionals; for example, by accessing relevant information from the Internet, mass media or alternative providers. Others may thrive on the basis of health-screening programmes that direct them to specific resources, delivered separately from routine care. Some patients may need single or small numbers of contacts with expert help. Others, particularly those with multiple problems, may need continuing help in finding their way through complicated situations. Some patients may be a long way, emotionally and practically, from thinking ahead, and may need time and support before such issues can be addressed. If the starting point lies in the future, the system must have the flexibility to wait, without losing contact.

Within the heterogeneity of individual needs and solutions, it is important to consider the issue of inequalities in health and health care (Watt, 2002), particularly when anticipatory care programmes are often attempting to reach people living in what are perceived to be 'hard-to-reach' deprived areas. A major challenge is to deliver anticipatory care in clinical settings where there may be neither the time, nor the resolve or the resources to move beyond reactive care (Mercer and Watt, 2007; Baum et al., 2009). The solution is not only the political task of finding extra resources; it is also knowing how best to use additional resources in a culture in which both patients and professionals have become used to expecting less.

\section{Interplay of theory and evidence}

A fully fledged system of anticipatory care would be grounded in theory and evidence concerning the nature of its active ingredients, programme pathways and the heterogeneity of individual needs and solutions. Although it would be ideal to posses such theory and evidence at the outset, this is not yet the case. It is important, therefore, to view intervention planning and knowledge building as an ongoing process.

Evaluations provide an opportunity to test current theory and to identify areas of the theory 
that need greater exposition (Mark et al., 2001). For example, it is rare for theories of complex interventions to explain and predict whether, where, how and for whom particular aspects of the intervention are likely to work. Evaluations provide a chance to understand such heterogeneity.

\section{Conclusion}

The theory and practice of anticipatory care are evolving. Evidence of 'what works' is important, but such evidence is patchy and usually based on small parts of the overall programme of anticipatory care. In such circumstances, the challenge is to maintain an overview of the programme, incorporating the heterogeneity of problems and solutions, so that progress and review are not dominated by the parts of the programme that are easiest to deliver.

We have described how anticipatory care can begin either as a separate new activity following a screening model, or in close association with conventional reactive care. To achieve high levels of population coverage, both approaches may be needed, but for different groups of people.

The concepts and practice of anticipatory care do not belong exclusively to general practice, family medicine, public health, health improvement or any single discipline. Successful programmes require joint working and shared leadership to provide the necessary combination of coverage, continuity, flexibility and co-ordination.

Irrespective of how and where anticipatory care starts, effective, equitable and sustainable delivery involves three challenges of integration. First, care should be integrated at the level of the patient, so that the focus of anticipatory care is firmly on the needs and experience of patients, rather than the interests and activities of providers. Second, available resources should be integrated in the service of a target population so that all continue to have the opportunity of being included. Third, all this effort should be integrated over time, so that promising starts are followed by sustainable, long-term productive contacts, relationships and outcomes.

Julian Tudor Hart's example was to combine these elements in the microcosm of a single general practice, using epidemiology to measure what he did and to show what could be achieved. For health-care systems round the world, facing problems of fragmentation, spiralling costs and increasing inequity, the gauntlet he threw down is to develop similarly integrated systems for the societies they serve.

His example is not a simple prescription to follow. Two decades after the publication of his 25-year review, this remains the only long-term example in the primary-care literature of a programme of comprehensive, long-term, anticipatory care. Surrounded by mountains, his population was easier to track over a long period than is the case in most urban populations. The type of leadership required to innovate within a small practice does not necessarily transfer to the challenges of joint working across professional and service boundaries. His levels of social commitment and conviviality were unusual.

It would be a mistake, however, to dismiss the example as exotic, and unworkable in ordinary circumstances. The basic elements of an unconditional patient-centred approach, building longterm relationships and maximizing population coverage are ubiquitous in many health-care systems. That they are seldom combined or geared to the level pioneered by Julian Tudor Hart is a challenge that can be overcome.

However, the internal strengths of general practice are not enough. They have to be combined with external resources and skills, introducing challenges of leadership and co-ordination that are not confined to working with colleagues in health improvement. The late US Senator Hubert Humphrey said,

the moral test of government is how that government treats those who are in the dawn of life, the children; those who are in the twilight of life, the elderly; and those who are in the shadows of life, the sick, the needy and the handicapped.

Young families, people approaching old age and patients with chronic conditions, especially those with multiple morbidity, are other starting points for anticipatory care, adding continuity, co-ordination and coverage to person-centred care. In each case, general practice provides a hub, but needs to work with other professionals and services.

Whether such collaboration and integration are achieved depends on many factors but crucially on the quality of relationships that are developed

Primary Health Care Research \& Development 2011; 12: 3-10 
over time, not only between patients and professionals, but also between professionals working together in the service of a local population. The challenge for everyone is to imagine, review, support and reward the sum of such efforts over long periods of time. Anticipatory care in these terms is the proving ground for health care in the 21st century.

\section{Acknowledgement}

We are pleased to thank Julian Tudor Hart for helpful comments on an earlier draft of this paper. The views expressed are those of the authors alone. COD is co-leading the national evaluation of 'Keep Well', a Scottish Government initiative designed to deliver anticipatory care to adults living in areas of socio-economic deprivation. SS led the evaluation of the 'Have a Heart Paisley' demonstration project. GW worked with Julian Tudor Hart at Glyncorrwg in the early 1980s.

\section{References}

Baum, F.E., Begin, M., Houweling, T.A.J. and Taylor, S. 2009: Changes not for the fainthearted: reorienting health care systems toward health equity through action on the social determinants of health. American Journal of Public Health 99, 1967-974.

Craig, P., Dieppe, P., Macintyre, S., Michie, S., Nazareth, I. and Petticrew, M. 2008: Developing and evaluating complex interventions: the new Medical Research Council guidance. BMJ 337, a1655.

Freeman, G.K., Olesen, F. and Hjortdahl, P. 2003: Continuity of care: an essential element of modern general practice. Family Practice 20, 623-27.
Hart, J.T. 1970: Semi-continuous screening of a whole community for hypertension. Lancet ii, 223-26.

Hart, J.T. 1982: Measurement of omission. BMJ 284, 1686-689.

Hart, J.T. 1993: Hypertension: community control of high blood pressure. Oxford: Radcliffe Medical Press.

Hart, J.T. 2006: Political economy of health care: a clinical perspective. Bristol: Policy Press.

Hart, J.T., Thomas, C., Gibbons, B., Edwards, C., Hart, M., Jones, J., Jones, M. and Walton, P. 1991: Twenty five years of case-finding and audit in a socially deprived community. BMJ 302, 1509-513.

Mark, M.M., Henry, G.T. and Junes, G. 2001: Evaluation: an integrated framework for understanding, guiding and improving policies and programs. San Francisco: Josey Bass.

Mercer, S.W. and Watt, G.C.M. 2007: The inverse care law: clinical primary care encounters in deprived and affluent areas of Scotland. Annals of Family Medicine 5, 503-10.

Murray, C.L. and Frank, J. 2008: Health metrics and evaluation: strengthening the science. Lancet 371, 1191-199.

Pawson, R. 2006: Evidence-based policy: a realist perspective. London: Sage publications.

Rose, G. 1985: Sick individuals and sick populations. International Journal of Epidemiology 14, 32-38.

Scottish Executive. 2005: Delivering for health. Retrieved February 2010 from http://www.scotland.gov.uk/Resource/ Doc/76169/0018996.pdf

Scottish Government. 2007: Better health, better care: action plan. Retrieved February 2010 from http://www.scotland. gov.uk/Resource/Doc?206458/0054871.pdf

Sridharan, S., Gnich, W., Moffat, V., Bolton, J., Harkins, C., Hume, M., Nakima, A., MacDougall, I. and Docherty, P. 2008: Evaluation of primary prevention intervention: have a Heart Paisley phase 2. Glasgow: NHS Health Scotland (http://www.healthscotland.com/documents/2881.aspx).

Stange, K.C. 2009: The problem of fragmentation and the need for integrative solutions. Annals of Family Medicine 7, 100-03.

Van den Dool, C. 1973: Allgemeinmedizin international 3, 100. Watt, G. 2002: The inverse care law today. Lancet 360, 252-54.

World Health Organization. 2008: The world health report 2008: primary health care now more than ever. Geneva, Switzerland: World Health Organization. 